\title{
IMPROVED SINGLE IMAGE DEHAZING BY FUSION
}

\author{
Nitish Gundawar ${ }^{1}$, V. B. Baru ${ }^{2}$ \\ ${ }^{I}$ ME Student, Department of Electronics and Telecommunication, Sinhgad College of Engineering, Pune, \\ ${ }^{2}$ Associate Professor, Department of Electronics and Telecommunication, Sinhgad College of Engineering, Pune,
}

\begin{abstract}
One of the major problems in image processing is the restoration of images corrupted by various types of degradations. Images of outdoor scenes often contain atmospheric degradation, such as haze and fog caused by particles in the atmospheric medium absorbing and scattering light as it travels to the observer. Although, this effect may be desirable from an artistic stand point, for a variety of reasons one may need to restore an image corrupted by these effects, a process generally referred to as haze removal. This paper introduces improved haze removal technique based on fusion strategy that combines two derived images from original image. These images can be obtain by performing white balancing and contrast enhancement operation. These derived images are weighted by specific weight map followed by Laplacian and Gaussian pyramid representations to reduce artifacts introduce due to weight maps. Unlike other techniques this approach requires only original degraded image to remove haze which makes it simple, straightforward and effective.
\end{abstract}

Keywords: Outdoor applications, fusion, dehazing, image pyramid

\section{INTRODUCTION}

Images of outdoor scenes often contain haze, fog, or other types of atmospheric degradation caused by particles in the atmospheric medium absorbing and scattering light as it travels from the source to the observer. Image obtained at other end is characterized by reduced contrast and faded colours. While this effect may be desirable in an artistic setting, it is sometimes necessary to undo this degradation. Weather conditions differ mainly in the types and sizes of the particles involved and their concentration in space. A great deal of effort has gone into measuring particle sizes and concentrations for a variety of conditions as shown in table I. For example, many computer vision algorithms rely on the assumption that the input image is exactly the scene radiance, i.e. there is no disturbance from haze. When this assumption is violated, algorithmic errors can be catastrophic. One could easily see how a car navigation system that did not take this effect into account could have dangerous consequences. Accordingly, finding effective methods for haze removal is an ongoing area of interest in the image processing and computer vision fields. This task is important in several outdoor applications such as remote sensing, intelligent vehicles, underwater imaging and many more.

In this paper improved fusion based haze removal technique is discussed. The main concept of fusion is to combine two or more images into single image that can be more suitable for some intended purposed [16]. Therefore, image fusion is effective technique that is designed to maximize relevant information into fused image.
Table-1: Weather conditions and associated particles types, sizes and concentration [2]

\begin{tabular}{|l|l|c|c|}
\hline Conditions & Particle Size & Radius $(\mu \mathrm{m})$ & $\begin{array}{l}\text { Concentration } \\
\left(\mathrm{cm}^{-3}\right)\end{array}$ \\
\hline Air & Molecule & $10^{-4}$ & $10^{-19}$ \\
\hline Haze & Aerosol & $10^{-2}-1$ & $10^{3}-10$ \\
\hline Fog & $\begin{array}{l}\text { Water } \\
\text { Droplet }\end{array}$ & $1-10$ & $100-10$ \\
\hline Cloud & $\begin{array}{l}\text { Water } \\
\text { Droplet }\end{array}$ & $1-10$ & $300-10$ \\
\hline Rain & Water Drop & $10^{2}-10^{4}$ & $10^{-2}-10^{-5}$ \\
\hline
\end{tabular}

The main idea behind fusion based dehazing technique is to combine images derived from degrade image. Two images are derived by performing white balance and contrast enhancement operation on original degraded image. This ensures the visibility in hazy and haze free region of image and also eliminate unrealistic color cast introduced due to atmospheric color. In fusion framework the derived inputs are weighted by three weight maps i.e. luminance, chromatic and saliency weight maps [1]. These weight maps ensure to preserve regions with good visibility. However, artifacts introduced by weight maps can be eliminated by fusing Laplacian pyramid representation of derived inputs and Gaussian pyramid representation of normalized weight that yields dehaze version of original degraded image.

The rest of the paper is structured as follows. Below in section 2 previous dehazing methods are briefly discussed. In section 3 theoretical aspects of light propagation is discussed. In 
section 4 details of fusion based dehazing method is presented. In next section experimental results analysis is performed based various parameters. In section 6 conclusion is highlighted and future work is predicted.

\section{LITERATURE REVIEW}

In many image processing and vision applications, enhancing and restoring images represents fundamental task. There are large numbers of dehazing methods and these existing methods can be grouped into several main classes.

Earlier haze removal techniques require multiple images of same scene or additional supplemental equipments. Methods [2], [3] include under this class. These methods improves visibility in restored image, but their main drawback is due to their acquisition step that in many cases is time consuming and difficult to carry out.

Another class of methods are polarization techniques [4]-[7], that are based on fact that airlight is partially polarized. By taking difference of two images of same scene under different polarization angles, it becomes possible to estimate the magnituide of polarized haze light. The methods include under this class have shown less robustness for scenes with dense haze where polarization light is not major degradation factor.

Another category of techniques assumes known model of scene [8]. These techniques employ an approximated depth map obtained after collecting information from several users about areas that are degraded or not by poor weather conditions. The Deep photo [9] is a more precise system since it uses the existing geo referenced digital terrain and urban models to restore foggy images. The depth information is obtained by iteratively aligning the 3D models with the outdoor images.

Restoration of images from single image is more challenging problem. Solutions for such cases have been introduced only recently [10]-[13]. These methods are roughly divided into contrast based and statistical based approaches. Tan [11] and Tarel [13] methods belongs to first category whereas, methods of Fattal [10], He [12], kartz [14] belong to second category.

Tan [11] observes that a haze free image must have higher contrast compared with the input hazy image and removes the haze by maximizing the local contrast of the restored image. The results are visually compelling but may not be physically valid. The contrast-based enhancing approach of Tarel [13] has shown to be computationally effective technique, but assumes as well that the depth-map must be smooth except along edges with large depth jump. Fattal [10] decomposed image into two components i.e. light which is reflected from surface (albedo) and shading, and then estimate the scene radiance based on independent component analysis (ICA) assuming that the shading and object depth are locally uncorrelated. The main drawback of this method is that it cannot handle heavy haze images. He [12] builds their approach on statistical observation of dark channel. In which object depth in hazy image is estimated based on dark channel prior, which assumes at least one color channel should have small pixel value in haze free image. To refine depth map of objects alpha matting performed. The dark channel prior may be invalid when the scene object is similar to airlight (e.g. snowy ground or white wall) also it requires additional post processing which leads to higher complexity. An attempt was made to remove haze effect from image using fusion principle in [15]. However, this approach require visible and near infrared (NIR) image of same scene in order to perform fusion. This approach is hard to carry out and hence it is time consuming.

\section{BACKGROUND: LIGHT PRPOGATION}

In case of outdoor applications it is not necessary that amount of light emitted by camera the same amount reflected back to camera. On the other hand, in almost every practical scenario light reflected from target object is scattered or absorbed in atmosphere before it reaches to camera. This happens due to presence of turbid medium in atmosphere which deflects light from original course of propagation as a result images with poor visibility and contrast are captured.

In computer vision, the optical model, which is widely used to approximate the image formation in bad weather condition, is as shown in figure 1. This model is commonly known as image degradation model or atmospheric scattering model proposed by McCartney. From figure1 it has been observed that captured image is represented by linear combination of two main components i.e. direct attenuation (D) and airlight (A) as describe in equation 1.

$$
\begin{aligned}
& \mathrm{I}(\mathrm{x})=\text { Direct attenuation }(\mathrm{A})+\operatorname{Airlight}(\mathrm{A}) \\
& \mathrm{I}(\mathrm{x})=\mathrm{J}(\mathrm{x}) \mathrm{T}(\mathrm{x})+\mathrm{A}(1-\mathrm{T}(\mathrm{x})) \ldots \ldots \ldots \ldots(1)
\end{aligned}
$$

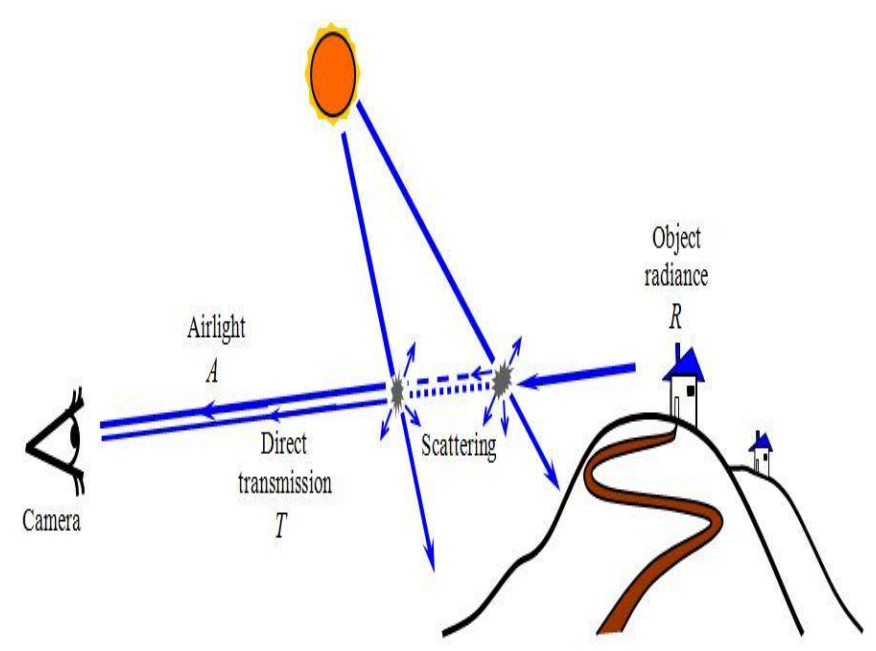

Fig-1: Image degradation model [1] 
The first component, i.e $\mathrm{D}(\mathrm{x})=\mathrm{J}(\mathrm{x}) \mathrm{T}(\mathrm{x})$ represents how the scene radiance is attenuated due to medium properties. The second component, i.e. $\mathrm{A}(\mathrm{x})=\mathrm{A}(1-\mathrm{T}(\mathrm{x}))$ represents the main cause of color shifting.

Where $I(x)$ is the observed intensity for each pixel $x, J(x)$ is the scene radiance (the original haze-free image to be recovered), $\mathrm{A}$ is the global atmospheric light, and $\mathrm{T}(\mathrm{x})$ is the medium transmission indicating the portion of light that is not scattered and reaches the camera. Assuming a homogenous medium, the transmission $\mathrm{T}$ is determined as

$$
\mathrm{T}(\mathrm{x})=e^{(-\beta d(x))}
$$

Where,

$\boldsymbol{\beta}$ is medium attenuation coefficient due to scattering $\mathrm{d}$ is distance between observer and the considered surface

\section{FUSION BASED DEHAZING}

This section presents the details fusion technique that employs only the inputs and weights derived from the original hazy image. The fundamental idea is to combine several input images (guided by weight maps) into single one, keeping only the most significant features of them. This technique performs following three steps in order to remove haze from degraded image.

Step1: Generation of two input images from original.

Step2: Defining weight measures.

Step3: Fusion of inputs and weight measures.

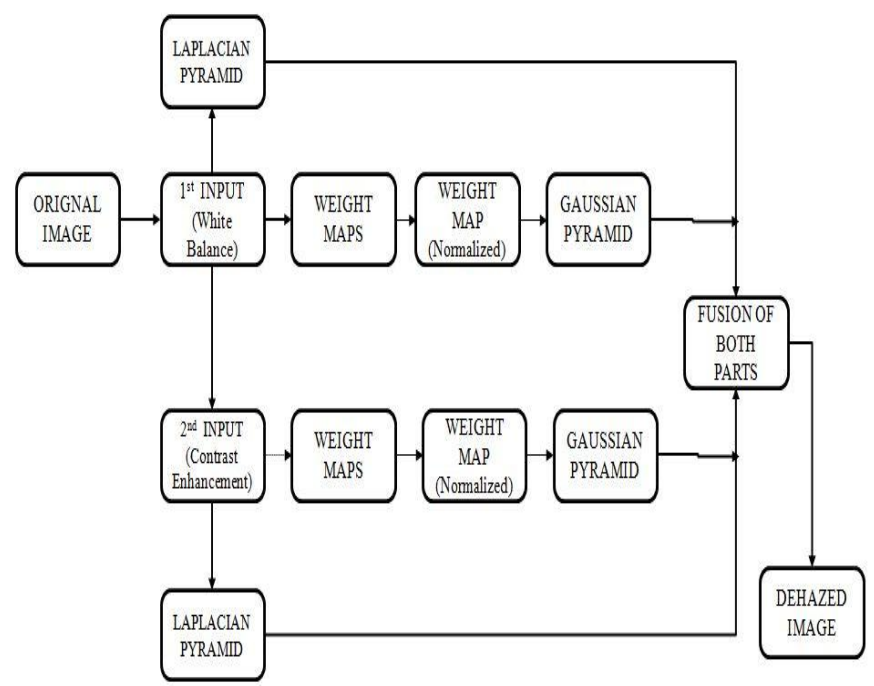

Fig-2: Flow of fusion based dehazing approach

\subsection{Definition of Inputs}

Fusion based dehazing approach takes two inputs derived from original image. The first input is obtained by performing white balance operation on original image. White balancing is an important processing step that aims to enhance the image appearance by discarding unwanted color casts, due to various illuminations. Nevertheless, white balancing solely is not able to solve the problem of visibility, and therefore we derive additional input in order to enhance the contrast of degraded image.

The second input is selected in order to increase contrast in those regions that suffers due to airlight influence. This can be done by performing either histogram equalization or gamma correction on first derived input as shown in figure 3 . It has been observed that this step significantly amplify the visibility in hazy part but on the other hand fine details of image get destroy. Therefore, in order to eliminate this degradation a proper weight maps are defined for each input.
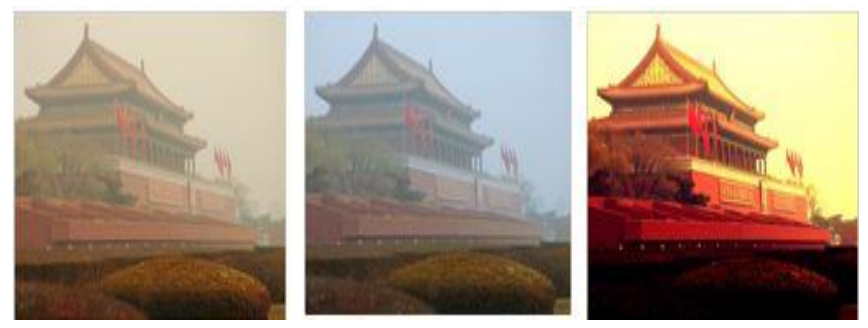

Original Hazy Image White Balance Contrast Enhancement

Fig -3: Derived inputs from original image

\subsection{Weight Measures}

The derived inputs are weighted by following three weight maps. These weight maps aim to preserve the regions with good visibility.

The Luminance weight map measures visibility of each pixel. It assigns high values to regions with good visibility and small values to the rest. Based on the RGB color channel information this weight map is processed. Luminance weight map is computed as the deviation between RGB color channel and luminance from input [1]. However, it is observed that this weight map reduce global contrast and color information. In order to overcome these effects two additional weight maps are defined: a chromatic map (color information) and saliency map (global contrast).

The Chromatic weight map is designed to control saturation gain in output image. This weight map is simply computed as distance between its saturation value and the maximum of saturation range [1]. Thus small values are assigned to pixels with reduced saturation while the most saturated pixels get high values. This weight map is motivated by the fact that human being generally prefers images that are characterized by high level of saturation.

The Saliency weight map identifies the degree of conspicuousness with respect to the neighborhood regions. 
Visual saliency is the perceptual quality that makes an object, person, or pixel stand out relative to its neighbors and thus, capture our attention [17]. Detection of visually salient image regions plays important role for applications like object recognition, object segmentation and adaptive compression. Following are some requirements for a saliency detector [17]:

- Maximizes the largest salient objects.

- Uniformly highlighted whole salient regions.

- Establish well-defined boundaries of salient objects.

- Discard high frequencies arising from texture, noise and blocking artifacts.

- Efficiently output full resolution saliency maps.

Figure 4 shows different weight maps for each derived input. It has been observed that impact of all these measures is equally important. However, the first measure has the highest impact on the visibility. The resulted weight is obtained by multiply all three weight maps. To yield consistent results resultant weight map is normalized.
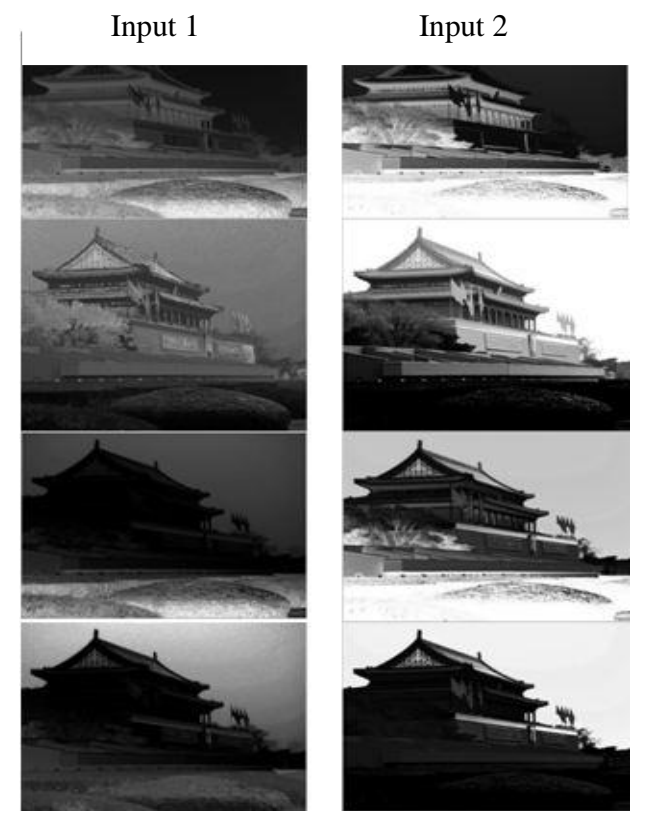

\section{Luminance}

Weight Map

Chromatic

Weight Map

Saliency

Weight Map

Normalized

Weight Map

Fig -4: Weight maps of derived

\subsection{Multi-Scale Fusion}

In practice, each input is decomposed into pyramid by applying Laplacian operator at different scales. Laplacian pyramid of image is obtained by applying band pass filter followed by down sampling operation [18]. As a band pass filter, pyramid construction tends to enhance image features such as edges, which plays important role for image interpretation. Each level in Laplacian pyramid represents the difference between successive levels of Gaussian pyramid. Similarly, for each normalized weight map Gaussian pyramid is estimated. The Gaussian pyramid is a sequence of images obtained by applying low pass filter followed by down sampling operation [18]. The Gaussian and Laplacian pyramid representation is as shown in figure 5. Now considering that both Gaussian and Laplacian pyramids have the same number of levels, mixing or fusion between Laplacian inputs and Gaussian normalized weight map is performed at each level independently yielding fused pyramid which is considered as dehazed version of original hazy degraded image as shown in figure 6 .

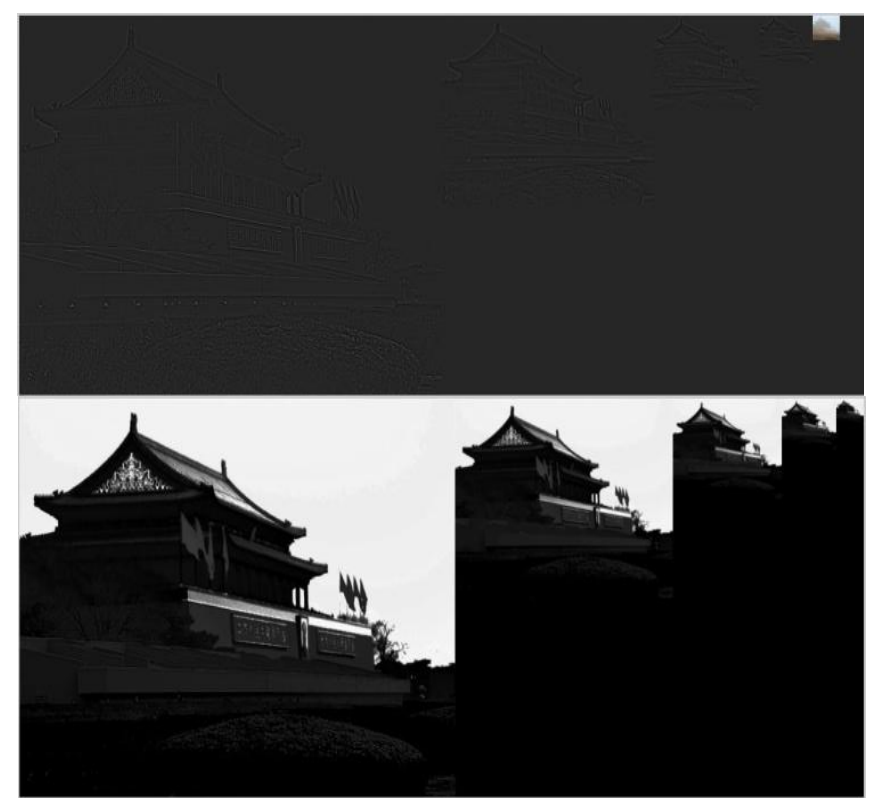

Fig-5: Laplacian and Gaussian Pyramid representation

Fusion based dehazing approach has several advantages over existing dehazing methods. First, it performs an effective perpixel computation, different from the majority of existing methods that processes patch. Secondly, complexity of this approach is lower than most of the previous strategy [10]-[14], as it is not necessary to estimate depth map. Finally, this approach performs faster, which makes it suitable for real time applications.

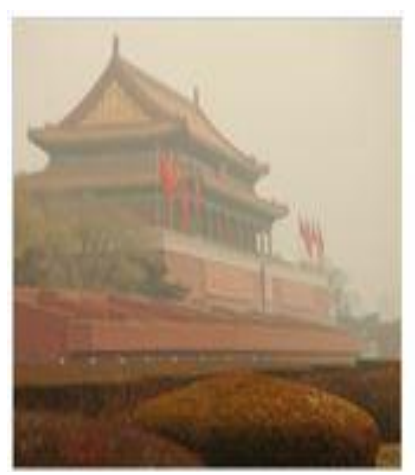

Orignal Hazy Image

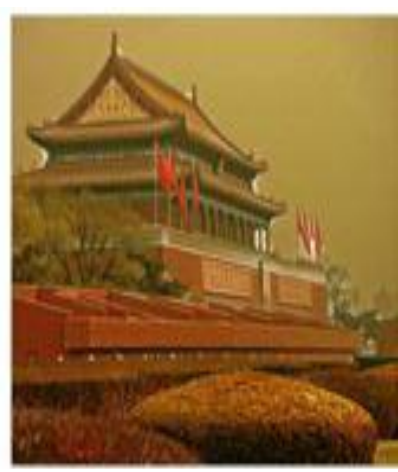

Final Dehazed Image
Fig-6: Final output 
Original Hazy Image
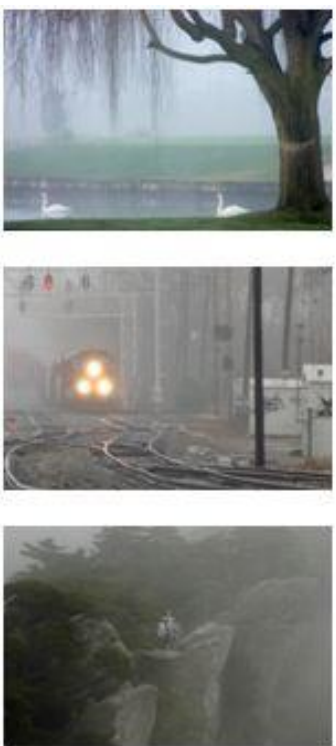

Fattal [10]
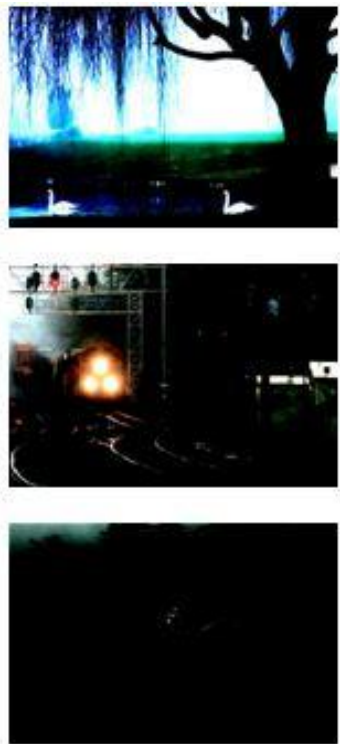

Tarel [13]
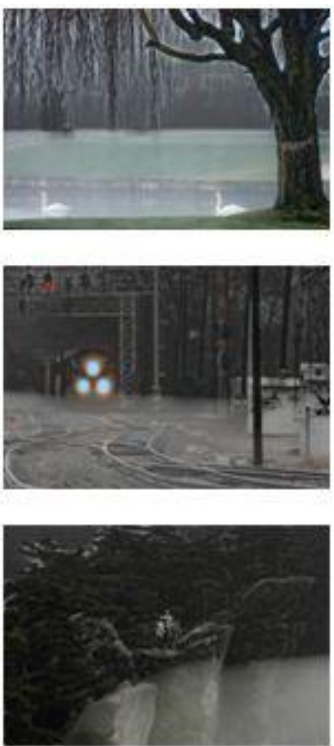

Fusion Based Dehazing [1]
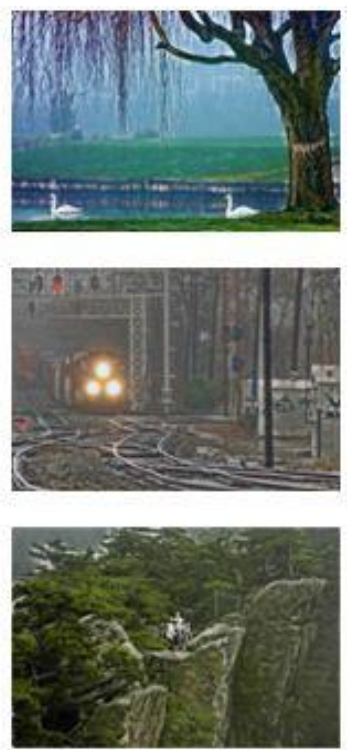

Fig-7: Comparison of Fattal [10] and Tarel [13] techniques with Fusion based dehazing

\section{EXPERMENTAL RESULTS}

To evaluate the performance of fusion based dehazing method, we apply this method to recover several hazy images and compare this method with the two popular existing single image based methods of Fattal [10] and Tarel [13]. In [10], an albedo estimation-based method was proposed whereas method in [13] based on contrast enhancement. In our experiment, we perform the method introduce in section III using MATLAB 12 on PC with a $2.20 \mathrm{GHz}$ Intel core2duo CPU. Figure 7 shows the comparative analysis of Fattal, Tarel and fusion based dehazing approaches.

In order to check the robustness of fusion based dehazing approach Peak signal to noise ratio and Mean square error is estimated and it has been observed that this approach outperform the other two single image based dehazing technique in [10],[13] as shown in table 2 and table 3.

Table-2: Comparison of PSNR

\begin{tabular}{|l|l|l|l|}
\hline Images & Fattal [10] & Tarel [13] & $\begin{array}{l}\text { Fusion based } \\
\text { approach [1] }\end{array}$ \\
\hline Img1 & 6.5947 & 5.5342 & 5.5162 \\
\hline Img2 & 11.829 & 4.484 & 4.3151 \\
\hline Img3 & 12.572 & 6.1993 & 6.1832 \\
\hline Img4 & 8.7858 & 3.4572 & 3.4525 \\
\hline Img5 & 9.1482 & 4.5801 & 4.5784 \\
\hline
\end{tabular}

Table-3: Comparison of MSE

\begin{tabular}{|l|l|l|l|}
\hline Images & Fattal [10] & Tarel [13] & $\begin{array}{l}\text { Fusion based } \\
\text { approach [1] }\end{array}$ \\
\hline Img1 & 14243 & 18183 & 18258 \\
\hline Img2 & 4257.6 & 23157 & 24675 \\
\hline Img3 & 2958.7 & 15584 & 15601 \\
\hline Img4 & 8600.1 & 29334 & 29365 \\
\hline Img5 & 7911.6 & 22650 & 22659 \\
\hline
\end{tabular}

The key advantage of this technique is that it does not necessary to estimate depth map, which reduces complexity to a great the extent. Moreover it has been observed that final output obtained is more pleasing than any other methods. Furthermore, compared with most of the existing techniques, an important advantage of fusion based dehazing is required computation time, which is able to process a 600x800 image in approximately 30-32 seconds as shown in table 4 .

Table-4: Comparison of computation time

\begin{tabular}{|l|l|l|l|}
\hline Images & Fattal [10] & Tarel [13] & $\begin{array}{l}\text { Fusion based } \\
\text { approach [1] }\end{array}$ \\
\hline Img1 & $167.59 \mathrm{sec}$ & $100.91 \mathrm{sec}$ & $30 \mathrm{sec}$ \\
\hline Img2 & $125.30 \mathrm{sec}$ & $107.82 \mathrm{sec}$ & $32 \mathrm{sec}$ \\
\hline Img3 & $116.56 \mathrm{sec}$ & $110.48 \mathrm{sec}$ & $31 \mathrm{sec}$ \\
\hline Img4 & $52 \mathrm{sec}$ & $41 \mathrm{sec}$ & $23 \mathrm{sec}$ \\
\hline Img5 & $75 \mathrm{sec}$ & $66 \mathrm{sec}$ & $24 \mathrm{sec}$ \\
\hline
\end{tabular}




\section{CONCLUSIONS AND FUTURE WORK}

The fusion based dehazing approach disussed in this paper can effectively restore image color balance and remove haze and fogg. This is first fusion dehazing approach that is able to solve such problems using only one degraded image. This technique is based on selection of appropriate weight maps and inputs, a fusion approach can be used to obtain dehazed version of hazy images. Moreover, it has been observed that this approach outperform the other single image based dehazing techniques. The method is faster than existing single image dehazing strategies and yields accurate results. In future work we would like to test this approach on underwater images and images from intelligent vehicles.

\section{ACKNOWLEDGEMENTS}

I wish to express my sincere thanks and deep sense of gratitude to respected mentor and guide Mr. V. B. Baru Associate Professor in Department of Electronics and Telecommunication Engineering of Singhgad College of Engineering, Pune for the technical advice, encouragement and constructive criticism.

\section{REFERENCES}

[1] C.O Ancuti and C. Ancuti, "Single image dehazing by multi-scale fusion," IEEE Trans. Image Processing, Aug.2013, vol.22, pp. 3271-3282.

[2] S. Narasimhan and S. Nayar, "Contrast restoration of weather degraded images," IEEE Trans. Pattern Anal. Mach. Intell., vol. 25, no. 6, pp. 713-724, Jun. 2003.[3] S. Narasimhan and S. Nayar, "Vision in bad wheather," in Proc. IEEE Int. Conf. Comput. Vis., Sep. 1999, pp. 820-827.

[3] S. Narasimhan and S. Nayar, "Chromatic framework for vision in bad weather," in Proc. IEEE Conf. Comput. Vis. Pattern Recognit., Jun. 2000, pp. 598605.

[4] T. Treibitz and Y. Y. Schechner, "Polarization: Beneficial for visibility enhancement" in Proc. IEEE Conf. Comput. Vis. Pattern Recognit., Jun. 2009, pp. 525-532.

[5] Y. Y. Schechner, S. Narasimhan, and S. Nayar, "Polarization-based vision through haze," Appl. Opt., vol. 42, no. 3, pp. 511-525, 2003.

[6] S. Shwartz, E. Namer, and Y. Schechner, "Blind haze separation," in Proc. IEEE Conf. Comput. Vis. Pattern Recognit., 2006, pp. 1984-1991.

[7] Y. Schechner and Y. Averbuch, "Regularized image recovery in scattering media," IEEE Trans. Pattern Anal. Mach. Intell., vol. 29, no. 9, pp. 1655-1660, Sep. 2007.

[8] S. Narasimhan and S. Nayar, "Interactive dewheathering of an image using physical models," in Proc. IEEE Workshop Color Photometric Methods Comput. Vis., Oct. 2003, p. 1.
[9] J. Kopf, B. Neubert, B. Chen, M. Cohen, D. Cohen-Or, O. Deussen, M. Uyttendaele, and D. Lischinski, "Deep photo: Model-based photograph enhancement and viewing," ACM Trans. Graph., vol. 27, no. 5, p. 116, 2008.

[10] R. Fattal, "Single image dehazing," ACM Trans. Graph., SIGGRAPH, vol. 27, no. 3, p. 72, 2008.

[11] R. T. Tan, "Visibility in bad weather from a single image," in Proc. IEEE Conf. Comput. Vis. Pattern Recognit., Jun. 2008, pp. 1-8.

[12] K. He, J. Sun, and X. Tang, "Single image haze removal using dark channel prior," in Proc. IEEE Conf. Comput. Vis. Pattern Recognit., Jun. 2009, pp. 19561963.

[13] J.-P. Tarel and N. Hautiere, "Fast visibility restoration from a single color or gray level image," in Proc. IEEE Int. Conf. Comput. Vis.,Sep.-Oct. 2009, pp. 22012208.

[14] K. Nishino, L. Kratz, and S. Lombardi, "Bayesian defogging," Int. J. Comput. Vis., vol. 98, no. 3, pp. 263-278, 2012.

[15] L. Schaul, C. Fredembach, and S. Süsstrunk, "Color image dehazing using the near-infrared," in Proc. IEEE Int. Conf. Image Process., Nov. 2009, pp. 1629-1632.

[16] P. J. Burt, K. Hanna, and R. J. Kolczynski, "Enhanced image capture through fusion," in Proc. IEEE Int. Conf. Comput. Vis., May 1993, pp. 173-182.

[17] R. Achanta, S. Hemami, F. Estrada, and S. Süsstrunk, "Frequency-tuned salient region detection," in Proc. IEEE Conf. Comput. Vis. Pattern Recognit., Jun. 2009, pp. 1597-1604.

[18] P. Burt and T. Adelson, "The Laplacian pyramid as a compact image code," IEEE Trans. Commun., vol. 31, no. 4, pp. 532-540, Apr. 1983.

[19] T. O. Aydin, R. Mantiuk, K. Myszkowski \& H.-S. Seidel.Dynamic range independent image quality assessment. In SIGGRAPH, ACM Transactions on Graphics (TOG), volume 27, pages 1-10, 2008. 